\title{
An analysis of evidence-based best practices in the public vocational rehabilitation program: Gaps, future directions, and recommended steps to move forward
}

\author{
Michael J. Leahy ${ }^{\mathrm{a}, *}$, Fong Chan ${ }^{\mathrm{b}}$, John Luic ${ }^{\mathrm{c}}$, David Rosenthal ${ }^{\mathrm{b}}$, Timothy Tansey ${ }^{\mathrm{b}}$, \\ Paul Wehman ${ }^{\mathrm{d}}$, Madan Kundu ${ }^{\mathrm{e}}$, Alo Dutta ${ }^{\mathrm{e}}$, Catherine A. Anderson ${ }^{\mathrm{c}}$, Roy Del Valle ${ }^{\mathrm{a}}$, \\ Susan Sherman ${ }^{\mathrm{a}}$ and Frederick E. Menz ${ }^{\mathrm{c}}$ \\ ${ }^{a}$ Michigan State University, East Lansing, MI, USA \\ ${ }^{\mathrm{b}}$ University of Wisconsin-Madison, Madison, WI, USA \\ ${ }^{\mathrm{c}}$ University of Wisconsin-Stout, Menomonie, WI, USA \\ ${ }^{\mathrm{d}}$ Virginia Commonwealth University, Richmond, VA, USA \\ e Southern University-Baton Rouge, Baton Rouge, LA, USA
}

Revised/Accepted July 2014

\begin{abstract}
As an epilogue to this special issue, this article provides a brief overview of the research undertaken by the Rehabilitation Research and Training Center on Evidence-Based Practice in Vocational Rehabilitation (RRTC-EBP VR) in Phase II of their studies, and what was learned from the comprehensive review of the literature (e.g., Fleming, Del Valle, Kim \& Leahy, 2013) and multi-state case studies in terms of promising practices in state VR agencies (Leahy et al., 2013). Gaps identified in EBP research that hinders the implementation of efficacious, effective, and efficient services based on the best scientific evidence are discussed, and finally, recommendations for future research directions and knowledge translation initiatives that will improve the effectiveness of VR service delivery practices are provided.
\end{abstract}

Keywords: Evidence-based practice, vocational rehabilitation, organizational behavior, management practice, promising best VR practices

\section{Introduction}

According to the United States Census Bureau, approximately 56.7 million people have a disability, representing approximately $19 \%$ of the civilian noninstitutionalized population (Brault, 2012). National

*Address for correspondence: Dr. Michael J. Leahy, Office of Rehabilitation and Disability Studies, Michigan State University, 463 Erickson Hall, East Lansing, MI 48824, USA. E-mail: leahym@ msu.edu. statistics indicate that only $18 \%$ of people with a disability age 16 years and older are employed, compared to $63.7 \%$ of persons without a disability (U.S. Department of Labor, 2012a; 2012b). Unemployment and underemployment problems facing people with disabilities have been made even more acute due to the global financial crisis of 2007-2008, with the effects of the crisis still being felt today. There is no question that the recession had a disproportionate impact on workers with disabilities, with the number of employed workers with 
disabilities declining at a rate of about two to three times that of workers without disabilities (Fogg, Harrington, $\&$ McMahon, 2011; Kaye, 2010). Even today, the unemployment rate of $12.9 \%$ for people with disabilities is still appreciably higher than the $6.1 \%$ rate for people without disabilities (U.S. Department of Labor, Office of Disability Employment Policy, 2014). As a result, this large number of people with disabilities excluded from the labor market constitutes a disproportionate number of the poor in this country (Atkins \& Giusti, 2005).

According to the World Health Organization (1995), the greatest cause of suffering on earth is poverty. Unfortunately, the gulf between the poor and the rich of the world is widening. In the United States alone, incomes for the highest-earning one percent of Americans skyrocketed 31 percent between 2009 and 2012, while the rest of the country grew an average of just 0.4 percent (Saez, 2013). Poverty and income inequality have direct and indirect effects on the social, mental, and physical well-being of individuals with and without disabilities. There is ample evidence to support that income inequality produces psychosocial stress, which leads to deteriorating health and higher mortality over time (Dutta, Gervey, Chan, Chou, \& Ditchman, 2008; Krause, Carter, Pickelsimer, \& Wilson, 2008; Murali \& Oyebode, 2014). Without a doubt, lack of employment opportunities excludes people with disabilities from full community inclusion and participation, stalls upward mobility, greatly affects their health-related quality of life, and subjective well-being. As such, participation in competitive employment and other meaningful work activities is considered a fundamental human right and crucial to the physical and psychological well-being of people with disabilities (Dutta et al., 2008).

The state-federal vocational rehabilitation (VR) program, which serves approximately one million individuals a year and spends approximately three billion dollars annually plays an instrumental role to assist individuals with chronic illnesses and disabilities achieve their independent living and employment goals (Dean, Pepper, Schmidt, \& Stern, 2013; Dutta et al., 2008; Martin, West-Evans, \& Connelly, 2010; U.S. Government Accountability Office [GAO], 2005). Over half of the people with disabilities have found employment in integrated settings after receiving VR services (Dutta et al., 2008). However, the average hourly earnings of all VR customers in competitive employment are only $52 \%$ of the general workforce (Rehabilitation Services Administration [RSA], 2008). Minorities receive only $80 \%$ of the services provided to European American consumers. In addition, employment, earnings, and the amount of purchased services received while in the state-federal VR program varied significantly by the consumer's disability types and other personal factors (GAO, 2005).

Clearly, the state-federal VR program needs to do a better job to improve employment outcomes and help more people with disabilities work their way into the middle class. In this era of empowerment, accountability, and constrained budgets, state VR agency administrators need to transform the business models of their agencies by adopting organizational innovations and harnessing advances in information and communication technologies to deliver outcomes to individuals, communities and society at large that are of significant value (Ditchman et al., 2013; Technology and Entrepreneurship Center at Harvard, 2010). As a corollary, rehabilitation professionals must provide people with disabilities the most effective psychosocial and vocational services and interventions by integrating the best scientific evidence, with clinical expertise and client perspectives, to help them find good-paying jobs with benefits consistent with their abilities and career interests (Chan et al., 2011; Leahy, Thielsen, Millington, Austin \& Fleming, 2009).

Recently, the evidence-based practice (EBP) movement has begun to significantly influence the professional practice of rehabilitation counseling. Evidence-based practice advocates that rehabilitation professionals should have an interest in delivering the best possible services to their consumers, based whenever possible on the best clinical practices available from the research evidence (Chan, Tarvydas, Blalock, Strauser, \& Atkins, 2009). Leahy et al. (2009) suggested that the emphasis of VR in the future will be on the meaning of research findings to practitioners and consumers in improving services, interventions, and employment outcomes for persons with disabilities, and translating and disseminating EBP that come from research efforts to the level of the organization that will affect and inform practice and policy.

The development of theory-driven or model-driven research to inform best practices in rehabilitation will undoubtedly be highly important as professionals in the field strive to improve the effectiveness of VR services and outcomes, especially for subpopulations of VR consumers with the poorest rehabilitation outcomes (Chan et al., 2009). In this special issue, we reported the findings of the four-state multiple case study (Maryland, Mississippi, Texas, and Utah) conducted by researchers at the Rehabilitation Research and Training Center for 
Evidence-Based Practice in Vocational Rehabilitation (RRTC-EBP VR) to identify evidence-based best VR practices, as well as organizational and cultural factors promoting best practices in the public vocational rehabilitation program. This comprehensive case study of promising VR practices spans a three-year period and represented the perspectives of 29 leaders and administrators, 56 mid-level managers and 469 counselors in four state VR agencies.

As an epilogue for this special issue, the purpose of this article is to provide a brief overview of what we have learned from our comprehensive review of the literature (e.g., Fleming, Del Valle, Kim \& Leahy, 2013) and our multi-state case studies (Del Valle et al., 2014; Leahy et al., 2013; Sherman et al., 2014) in terms of promising practices in state VR agencies; identify the gaps in EBP research that hinders the implementation of efficacious, effective, and efficient services based on best scientific evidences; and make recommendations for future research directions and knowledge translation efforts that will improve the effectiveness of VR service delivery practices.

\section{Evidence-based practices in vocational rehabilitation}

There is a general misperception among stakeholder groups in the disability and rehabilitation communities (e.g., policymakers, advocates, and consumers) that rehabilitation counselors in state VR agencies do not use empirically validated interventions to improve the psychosocial, health, and employment outcomes of people with disabilities (Chan et al., 2011). To the contrary, many services provided by rehabilitation counselors in state VR agencies are supported by strong scientific evidence, including best practices in: (a) counseling, (b) skills training, and (c) supported employment (Chan et al., 2011). Each of these areas will be briefly discussed below, including some of the empirical evidence that is available on the effectiveness of these VR interventions and the overall effect of VR as an employment intervention for people with chronic illnesses and disabilities.

\subsection{Counseling and the working alliance between the counselor and consumer}

Psychological and vocational counseling is a major job function and knowledge domain of rehabilitation counselors (Leahy, Chan, Sung, \& Kim, 2013) and counseling and guidance is one of the most frequently provided state VR services (Ditchman et al., 2013). Wampold (2001) in his review of multiple meta-analyses concluded that the effect size $(d)$ of absolute efficacy of counseling/psychotherapy is relatively large and falls within the range of .75 to .85 . $\mathrm{He}$ indicated that a reasonable and defensible point estimate for the efficacy of counseling/psychotherapy would be 0.80 , which means that the average treated person does better than $79 \%$ of untreated persons (Wampold, 2001). In recent years, several state VR agencies have begun to provide motivational interviewing as in-service training for rehabilitation counselors to help them improve working relationship with consumers (Manthey, Jackson, \& Evans-Brown, 2011). Horvath, Del Re, Flückiger, and Symonds (2011) analyzed 190 studies representing over 14,000 consumers to examine the relation between the alliance in individual therapy and treatment outcome. They found an overall effect size of $r=.28(d=0.57)$ indicating that the working alliance has a moderate effect on counseling/psychotherapy outcome. And the working alliance, along with therapist effects, is one of the strongest validated factors influencing counseling and therapy success (Wampold, 2001).

\subsection{Skills training}

Promoting general efficacy and career self-efficacy is central to the mission of state VR agencies (Ditchman et al., 2013). Labor market training (e.g., vocational training, on-the-job training, job readiness training, and job seeking skills training) is an effective VR intervention especially for people with sensory disabilities, physical disabilities, and chronic illnesses. Bolton and Akridge (1995) published a meta-analysis of 15 studies examining the efficacy of a range of skills training (e.g., relationship skills, problem solving skills, and occupational choice skills) for VR consumers. They found that outcome measures resulted in an effect size of $d=0.93$, suggesting substantial benefit to the typical participant of state VR services. Rosenthal, Hoyt, Ferrin, Miller, and Cohen (2006) re-analyzed Bolton and Akridge's (1995) study using several alternative analyses to enhance and extend their conclusions. Rosenthal et al. (2006) obtained a more conservative effect size estimate of $d=0.79$, indicating that at the conclusion of treatment, VR consumers in the intervention group scored, on average, nearly four fifths of a standard deviation higher than consumers in the no treatment control group. 


\subsection{Supported employment}

For consumers with intellectual disabilities/developmental disabilities (ID/DD) and people with severe mental illness, supported employment and the individual placement and support (IPS) model of supported employment have been utilized as an effective and evidence-based vocational intervention. Recently, Wehman, Chan, Ditchman, and Kang (2014) examined the effect of supported employment intervention on the employment outcomes of transition-age youth with ID/DD (between 16 and 25 years old at the time of application) served by state VR agencies using a case-control study design. The classification and regression tree (CART) method was used to estimate propensity scores and to adjust for selection bias on the basis of prominent demographic covariates relevant to the dependent variable (i.e., propensity to receive supported employment). They found that social security beneficiaries who had received special education in secondary school had the highest propensity to receive supported employment as part of their VR services. Of the individuals in this homogeneous group who received supported employment as a VR intervention, 58\% obtained successful employment closures compared to the $37 \%$ employment rate of those who did not receive supported employment, $\chi^{2}$ $(1, N=3,687)=155.39, p<.001, d=0.42$. High school (regular education) graduates with intellectual disability or autism who were social security beneficiaries at the time of application also had a high propensity to receive supported employment as a VR intervention and those who received supported employment had a significantly higher employment rate (63\%) compared to those who did not received SE $(43 \%), \chi^{2}$ (1, $N=1,760)=68.87, p<.001, d=0.40$. The effect sizes of 0.40 and 0.42 for these two subgroups with high propensity to receive supported employment indicated that supported employment services provided by state VR agencies have a moderate effect on employment outcomes for people who received special education in high school and for individuals with ID/DD, especially for those who were social security beneficiaries.

Similarly, Campbell, Bond, and Drake (2011) conducted a meta-analysis to evaluate the effect of four randomized controlled trials comparing the IPS model of supported employment to traditional vocational interventions for people with severe mental illness. They reported relatively large effect sizes favoring IPS across all outcomes for people with severe mental illness: (a) 0.96 for job acquisition, (b) 0.79 for total weeks worked, and (c) 0.74 for job tenure. Recently, Dean, Pepper, Schmidt, and Stern (2013) evaluated the effect of training (by including supported employment as part of the training variable) with a cohort of individuals with cognitive impairments who applied for VR services in Virginia in state fiscal year 2000 (July 1, 1999 to June 30,2000 ) using an elaborate econometric returnon-investment model, they reported that holding other VR services constant, training significantly increases employment propensity (.36 in the short run and .13 in the long run) of people with cognitive impairments.

\subsection{Overall effect of VR interventions}

Although it is difficult to design a true experimental study to investigate the overall effect of state VR services because random assignment of consumers to treatment and control groups is not always possible as consumers who are eligible for services must be served immediately (Bolton, 2004), several researchers have attempted to evaluate the overall effect of VR on employment outcomes of people with disabilities using alternative strategies. For example, O’Neil, Mamun, Potamites, Chan, and Cardoso (2014) conducted a carefully designed case control study to examine the effect of state VR agency services on return-to-work outcomes of Social Security Disability Insurance (SSDI) beneficiaries using two administrative data sets that were matched prospectively — the longitudinal Disability Analysis File (DAF) formerly known as the Ticket Research File (TRF) from the Social Security Administration and the United States Department of Education, Rehabilitation Services Administration (RSA) Case Service Report File (RSA-911). Social Security Disability Insurance beneficiaries $(N=17,369)$ who accessed state VR agency services in 2000 were matched with SSDI beneficiaries who had never applied for state VR services based on 36 demographic covariates. Propensity score matching led to balance between the treatment and control groups on 34 of the 36 variables. Logistic regression analysis results revealed the treatment groups completing the trial work period at significantly greater rates than the control groups in the 10 -year period of the study.

Dean, Pepper, Schmidt, and Stern (2014) examined the association between the receipt of VR services and SSI/SSDI using a special panel dataset on Virginians with disabilities that applied for state VR services in 2000. They found that VR services are associated with lower rates of participation in disability insurance programs (a two-point drop in SSDI receipt and one-point 
drop in SSI receipt); VR service receipt is associated with lower take-up rates of SSI/ SSDI; and social security beneficiaries receiving substantive VR services are more likely to be employed.

\section{Promising practices identified in the four-state multiple case study}

In the RRTC-EBP VR multi-state case studies, we identified promising organizational management practices and clinical service delivery practices that are used by state VR agencies to improve the performance of their agencies and the employment outcomes of individual with disabilities (Del Valle et al., 2014; Leahy et al., 2013; Sherman et al., 2014). While the state-VR agencies that participated in the study promote promising practices at all levels of the organization, the omission of best practice measurements or detailed program evaluation designs for these interventions presents a substantial limitation in the movement toward the development and implementation of innovations and EBP, at either the organizational or service delivery level. As noted by Leahy et al. (2013), although these practices are highly promising in terms of staff and management perspectives, they generally lack empirical support at the level needed to refer to them as evidence-based practices. Also, while some of these practices were well documented, many did not meet the expectations of standardized and manualized interventions or services that are useful in replication efforts to examine portability and further validation. In the next section, we will highlight some of the most significant findings in relation to organizational/management and clinical service delivery promising best practices that were found in the multi-state agency sample in our study (see Table 1 for a synopsis of these promising best practices).

Table 1

Promising practices being used by state VR agencies

\begin{tabular}{l}
\hline I Organizational; and Management Best Practices \\
1. Advocacy culture that leads to innovation. \\
2. Outcome focused business models \\
3. Advanced information technology \\
4. Collaborative partnership that enhance results \\
5. Staff training and development \\
II. Clinical Service Delivery \\
1. Transition from School to Adulthood \\
2. Individual placement and Supported Employment Model \\
3. Worklace Specialized Skills Training \\
4. Benefits Counseling \\
5. Workplace Supports
\end{tabular}

\subsection{Organizational and management best practices}

Based on the findings of Sherman et al. (2014), it is clear that leadership style and management practice have a significant influence on whether innovations and evidence-based practices will be embraced and implemented in state VR agencies. Sherman and her colleagues (2014) found that the likelihood of engaging in innovative best practices is affected by several organizational and environmental factors such as culture, leadership, support for innovative and promising practices, partnerships, staff training and development, the working alliance and client-centered services. Specifically, leadership plays a significant role in the success of a state VR agency. Successful leaders foster the development of an organizational culture that encourages innovation at all levels and creates a supportive learning environment leading to sustainable synergy (Sherman et al., 2014).

Adhocracy culture. In state VR agency settings, leaders who foster a culture of adhocracy that facilitates a learning organization are the key to building a high performance VR agency. This is because adhocracy culture is externally focused and the emphasis is on flexibility or dynamism, adaptability, and change. Decision-making is decentralized and creativity, entrepreneurship, and risk-taking are expected and valued. In this culture, teams are formed around projects and communication flows up the chain of command. As a result, leaders and managers in state VR agencies that foster a culture of adhocracy highly value innovations from their staff and encourage building partnerships with external stakeholders (Sherman et al., 2014).

Outcomes-focused business models. Successful VR agencies also emphasize the use of outcomes-focused business models by adopting organizational innovations and harnessing advances in information and communication technologies to deliver outcomes that individuals, communities and society at large value. Leaders in high performance VR agencies value the importance of setting proper expectations, establishing specific goals, developing explicit plans for achieving these goals, deploying resources where needed, specifying accountability, and rewarding performance. Agencies that foster a culture of high expectations also emphasize the use of advanced information technologies in rehabilitation, they are highly datadriven, and actively use data to establish goals, to allocate resources, and monitor performance. Staff members across all levels of the organization including 
counselors, managers, and leaders are held accountable for achieving the objectives of the organization.

Advanced information and communication technologies. In the era of the Affordable Care Act, the use of health information technology to improve healthcare efficiency and outcome is expected. Similarly, state VR agencies that use outcome-focused business models are adopting organizational innovations and harnessing advances in information and communication technologies to deliver outcomes. For example, one agency in the multi-state case study is using web-based cloud technology to help manage business relations and assist staff in their efforts to bring job-ready consumers and businesses together. Another agency utilizes the "SharePoint," a Microsoft web-based cloud site, as a central site for staff members to post and share ideas around "best practices" as well as lessons learned.

Partnerships. Successful VR agencies value relationships with all partners including legislators, employers, community-based rehabilitation organizations, consumer organizations, and the general public. These agencies work closely with all stakeholder groups to ensure that each groups' needs are met through collaborative working relationships, seamless service delivery, and technical assistance. Inter and intra-agency partnerships are considered a critical component of service provision. Employers and individuals with disabilities are viewed as valued customers for agencies that use a dual-customer approach to match and prepare consumers for jobs that employers want to fill. These agencies also expend concerted efforts to promote awareness of VR, the people served, and the benefits of state VR services for both individuals with disabilities and the community.

Staff training and development. Training is essential to introducing innovations and support agency staff to implement innovations, including promising management practices and evidence-based VR practices. Successful VR agencies consistently emphasize the importance of having a highly trained staff and allocate resources to train their staff to implement innovative and promising practices aimed at enhancing service delivery and employment outcomes for agency customers (e.g., the Individual and Placement Support [IPS] model of supported employment, motivational interviewing, secondary transition, demand-side employment, and multicultural rehabilitation counseling).

Rapid services and quality control of support agencies. Successful VR agencies are expected to provide services to their consumers rapidly to help maintain motivation to work and engagement in VR services by maintaining a strong working relationship with and oversight of community-based rehabilitation organizations and other vendors that provide support services for VR agencies. They also establish concrete criteria and performance standards to screen support service agencies and the qualification of their personnel. Successful VR agencies that have good oversight and coordination with their support service agencies to guarantee rapid services for consumers are able to reduce the average time in rehabilitation and greatly improve employment outcomes.

\subsection{Clinical service delivery best practices}

Del Valle et al. (2014) in their qualitative analysis of the multi-state case study data identified a set of promising VR interventions that rehabilitation counselors utilize to improve the effectiveness of VR service delivery. The findings from the multiple case study analysis provided the beginnings of an understanding of the specific best practices implemented by state VR agencies to serve persons with disabilities seeking employment. The researchers identified 29 agency practices, subdivided into promising organizational practices (15 practices) and promising service delivery practices (14 practices) that were provided in varying degrees within the state VR agencies involved in this study. We will highlight below a few of the most promising practices identified that have empirical support.

Transition. Secondary transition services have received considerable attention among special educators and rehabilitation counselors as an important intervention for helping high school students with disabilities build pathways to postsecondary employment success. Transition is the process students and their families use to think about life after high school, to identify desired post school outcomes, and to plan community and school experiences to assure that the students acquire the knowledge and skills to achieve their postsecondary education and employment goals. Maryland VR developed the Maryland Seamless Transition Collaborative to optimize interagency collaboration and to provide continuous transition services to high school students with disabilities using the Guideposts for Success framework developed by the National Collaborative for Workforce and Disability/Youth. This five-year demonstration project demonstrated the effectiveness of VR-based transition services with $64 \%$ of the participants exiting school with a job or enrolling in postsecondary education. 
Individual placement and support (IPS) model of supported employment. People with severe mental illness represent one of the subpopulations of VR consumers with the lowest employment outcomes. As mentioned, IPS is an evidence-based practice that was developed to help promote the recovery of people who have severe mental illness through work (Campbell et al., 2011; Substance Abuse and Mental Health Services Administration, 2009). Vocational rehabilitation agencies in our case study have provided extensive training for their counselors to provide or coordinate IPS services for people with severe mental illness receiving services in their agencies, resulting in better employment outcomes for people with severe mental illness.

Workplace socialization skills training. Workplace socialization skills are a critical component of worker productivity influencing workers' ability to obtain and sustain competitive employment (U.S. Department of Labor, 1991). Mississippi VR counselors use the Smart Work Ethics soft skills training curriculum to teach consumers social skills and other soft skills required to function effectively in the workplace with encouraging results.

Benefits counseling. Work incentive planning and benefits services have been used by state VR agencies to successfully reduce the effect of disability benefits on discouraging people with disabilities from seeking employment or returning to work. The availability of benefits counselling services help to allay rehabilitation counselors' ambivalence about working with SSI/SSDI recipients and provide a means for counselors and consumers to work collaboratively to make informed decisions about work and how much work a consumer chooses to do, resulting in higher employment rates for social security beneficiaries.

Workplace support services. It is well documented in demand-side employment research that employers are more likely to hire a person with a disability with prior experience as a temp worker or an intern in the company (Chan et al., 2010). Similar to supported employment for people with ID/DD and psychiatric disabilities, embedded training has been successfully used by one VR agency in our study as a marketing tool and method to obtain employer buy-in for placing and hiring people with sensory and physical disabilities. Consumers who need workplace support are placed in an employment setting and received training along with supportive services needed to help consumers develop physical stamina and learn work skills. Once the training is completed, successful participants are hired by the host company or are placed in employment within the same industry.

\section{Gaps and future research directions}

After the multi-state study was completed the research team met to review, analyze, and discuss all of the findings in order to specifically identify the most critical gaps, future directions and research recommendations as important next steps in moving forward with evidence-based best practices in the public vocational rehabilitation program. These gaps and research recommendations are provided for three distinct areas that include: (a) organizational and management practices; (b) evidence-based VR best practices; and (c) knowledge translation. Each of these areas will be discussed with a particular focus on gaps and recommendations for future research.

\subsection{Organizational and management practices}

According to experts at Harvard University's Leadership for a Networked World (LNW), human service organizations must develop new competencies capable of responding in new and different ways to create and deliver outcomes in the "New Normal" global economy of slow GDP growth (Oftelie, 2011). They developed the Human Services Value Curve framework to help conceptualize how human service organizations need to do to transform their business models. The Human Services Value Curve comprises four stages of organizational maturation (regulative, collaborative, integrative, and generative); a description of the stages of organizational maturation is presented in Table 2.

A recent self-assessment survey conducted by the American Public Human Services Administration (APHSA, 2013) indicated the majority of health and human service agencies are generally between the first and second levels of maturity. The APHSA report concluded that public health and human service agencies, including state VR agencies, must be encouraged to move beyond the use of the basic regulative/collaborative business model and move towards an integrative/generative business model (APHSA, 2013; Technology and Entrepreneurship Center at Harvard, 2010). Using an integrative/generative business model, state VR agencies will be able to design, produce, market, deliver and support the delivery of VR services faster, leaner and in "flatter" organizational structures.

It is apparent from the results of our multi-state case studies that state VR agencies that are able 
Table 2

The four stages of organizational maturation

\begin{tabular}{|c|c|}
\hline Stage of organizational maturation & Description \\
\hline Regulative business model & $\begin{array}{l}\text { This model allows health and human service agencies to comply with program requirements. Programs } \\
\text { and processes are developed and managed according to discrete funding sources. Information } \\
\text { technology and support tools are designed to support program-specific management, funding, } \\
\text { eligibility, care management, and consumer interactions. }\end{array}$ \\
\hline Collaborative Business Model & $\begin{array}{l}\text { Human service agency expands beyond program "silos" and categorical management to help their } \\
\text { consumers receive an optimum mix of services that address their near-term and mid-term needs. }\end{array}$ \\
\hline Integrative Business Model & $\begin{array}{l}\text { Broadens its focus to the integration of multiple programs and services in order to improve consumer } \\
\text { service, increase participation, and support data-driven policy and decision-making. Advanced health } \\
\text { information technologies are used to support both back-office processes and front-office innovations } \\
\text { such as individualized client services focused on improving health outcomes, economic } \\
\text { self-sufficiency, and social inclusion. }\end{array}$ \\
\hline Generative Business Model & $\begin{array}{l}\text { Organizations expand to address complex human problems, socioeconomic issues, and opportunities } \\
\text { using extensively cross-boundary data and information to generate long-term individual and } \\
\text { community success. In practice, the culture, managerial and operational processes, and technology of } \\
\text { the organization will be highly adaptive and modular to enable multiple programs and institutions to } \\
\text { build, share, and deploy information and services on a rapid, ongoing and evolving basis. }\end{array}$ \\
\hline
\end{tabular}

to make proactive changes to respond to the economy, technology, political and social factors affecting human service agencies in today's do-more-with-less environment will produce better results than agencies that are content with the status quo. The agencies in the four-state sample have all moved from hierarchal to adhocracy cultures and created learning organizations that encourage staff at all levels to be innovative, to take advantage of advanced information technology, and employ effective best practices. These changes include eliminating the layers of bureaucracy, encouraging innovations, supporting a learning environment, emphasizing accountability and outcome-orientation, building partnerships with external stakeholders, and harnessing advances in information and communication technologies to deliver outcomes (i.e., responsiveness, efficiency, customer service, and good job with benefits for people with disabilities) that individuals, communities and society value. Although some of the multi-state case study findings related to leadership styles and management practices may be agency specific, the majority of the findings can be adopted or adapted by any state VR agency to improve organizational structure and the culture of the agency.

\subsection{Gaps and research directions in organizational and management practices}

We have not used a rigorous quantitative research design to establish the cause-effect relationship between the leadership styles and management prac- tices and employment success of consumers served by the state VR agencies in the multi-state case studies. The National Institute on Disability and Rehabilitation Research (NIDRR) has adopted the stages-of-research and stages-of-development frameworks to enhance NIDRR's efforts to generate evidence-based policies, practices, and products. These stages include exploration, intervention development, intervention efficacy, and scaled-up evaluation. Recommendations for filling the gaps in organizational research to improve organizational performance and effectiveness of state VR agencies are summarized in Table 3 .

Health and human service business model. In the terms of the research gap, at the exploration research stage, there is a need to convene a panel of scholars, policymakers, and administrators representing business and management, human services administration, vocational rehabilitation, and information technology fields. The calibre of the committee members should be similar to criteria used for reports commissioned by the Institute of Medicine. The expert panel/committee will be tasked to identify essential features of an integrative/generative business model optimized for effective leadership and management practices in state VR agencies. The features identified can be used to develop a fidelity measure for assessing factors ranging from leadership and governance to the use of technology, the efficiency of current workflows, and service delivery. The fidelity survey will be modelled after the SelfAssessment Survey used by the American Public Human Services Administration to study the extent human service agencies has been using the integrative/generative business model to improve organizational performance. 
Table 3

Recommendations for filling the gaps in VR organizational research

Organizational research collaborative/generative business practice in VR
Description

- Convene a panel of experts to identify essential features of an integrative/generative business model optimized for effective leadership and management practices in state VR agencies.

- Develop a fidelity measure for assessing factors ranging from leadership and governance to the use of technology, the efficiency of current workflows, and service delivery.

- Administer the fidelity measure as a self-assessment measure to determine the extent each agency's business model is similar or dissimilar to the integrative/generative business model (i.e., organizational maturity levels related to the Human Services Value Curve) and investigate the effect of maturity levels on organizational performance metrics including retention of talent, diversity, staffing and learning, employee engagement, productivity and efficiency, return on investment, and employment outcomes for people with disabilities.

- Develop and validate technical assistance, consultation and training interventions to assist state VR agencies move away from a regulative/collaborative business model and move toward an integrative/generative business model.

2. Develop and validate a brief measure to assess the - Investigate factors influencing propensity to adopt innovation among employees propensity of VR personnel to adopt innovations representing the entire continuum of the diffusion of innovation (i.e., innovators, early adopters, early majority, late majority, and laggards) in the agency.

- Conduct research to evaluate the efficacy of different social-cognitive interventions that can be used to secure buy-in for all adoption groups.

3. Develop and validate a brief measure of technology acceptance in state VR agencies

- Conduct research using a hybrid diffusion of innovation and technology acceptance model (TAM) to evaluate factors influencing the propensity of members of the VR organization to accept the use of advanced information and communication technologies as productivity tool and to improve service delivery and employment outcomes for people with disabilities.

4. Conduct research to improve talent management and retention
- Organizational research effort should be expanded to examine the relationship among work motivation, workplace stress, burnout, organizational commitment, work engagement, and job satisfaction and life satisfaction of employees in state VR agencies.
Each item in the VR agency self-assessment survey will be tied directly to a feature of the integrative/generative business model for state VR agencies. This selfassessment measure can be administered to all state VR agencies to determine the extent each agency's business model is similar or dissimilar to the integrative/generative business model (i.e., organizational maturity levels related to the Human Services Value Curve framework).

It is hypothesized that agencies that are farthest along the maturity path of implementing the integrative/generative business model will perform better on all important organizational performance metrics including retention of talent, diversity, staffing and learning, employee engagement, productivity and efficiency, return on investment, and employment outcomes for people with disabilities. If the fidelity measure was found to predict state VR agencies' organizational performance metrics, technical assistance, consultation and training interventions can be developed (intervention development) and validated (intervention efficacy) to help state VR agencies move away from a regulative/collaborative business model and move toward an integrative/generative business model, enabling these agencies to adopt organizational innovations and harness advances in information and communication technologies to deliver outcomes that are customized and responsive to individuals, communities and society want.

Diffusion of innovation. Adopting organizational innovations to deliver outcomes effectively is the hallmark of the integrative/generative business model. According to Rogers (2003), the propensity of members of an organization to adopt innovations can be classified into five groups according to the amount of time passing from innovation availability to adoption: (a) innovators $(2.5 \%)$, (b) early adopters $(13.5 \%)$; (c) early majority $(34.0 \%)$, (d) late majority $(34.0 \%)$, and laggards $(16.0 \%)$. In order to innovate and to solicit and secure top-to-bottom organization buy-in from the executive leadership to frontline staff (e.g., the decision to use smartphone technology to deliver workplace support service for consumers), it is important to identify subgroups of employees who are most receptive to innovations and can quickly incorporate the needed changes in their normal workaday routine as well as employees who are most resistive to change. For example, recruiting innovators and early adopters to engage in 
a novel evidence-based VR practice can maximize the chance that this EBP will be successfully implemented and its benefits demonstrated. Identifying counselors who are laggards or late majority to provide intensive training and supports to increase outcome valency on the use of the innovative practice can lead to complete buy-in from all frontline staff in the agency. Therefore, research should be conducted to develop and validate an effective diffusion of innovation measure in VR organization and to study factors influencing propensity to adopt innovation among employees representing the entire continuum of the diffusion of innovation in the agency. In addition, research should be conducted to evaluate the efficacy of different interventions that can be used to secure buy-in for all adoption groups.

Technology acceptance. Harnessing advances in information and communication technologies to deliver outcomes is a key feature of the integrative/generative business model. Technology is probably the most significant change agent in the world today and the powerful forces of social, mobile, cloud and information will continue to shape how we communicate, collaborate, and engage with people at all levels and across disciplines within organizations. Instead of relying on old legacy business systems, employees in business as well as human service organizations are expected to increasingly do most of their business from laptop, tablet, and smartphone using cross-device software; organizations are beginning to integrate information from social media including Facebook, LinkedIn, and Twitter; cloud storage systems are used to enable employees to access information anywhere; business and human organizations are providing employees with third-party apps and proprietary apps to boost productivity and meet client demands; employers are increasingly using online educational resources to help employees meet their career development and training goals.

As health and human service agencies become more consumer-centric instead of professional-centric, it is important for health and human service professionals to provide services in line with the customers' expectations including the use of advanced health communication technologies. Researchers have been using a hybrid diffusion of innovation and technology acceptance model (TAM) to study diffusion of proven information technologies for health care workers (de Veer, Fleuren, Bekkema, \& Francke, 2011). It is imperative to conduct research using TAM to evaluate factors influencing the propensity of VR counselors and supervisors to accept the use of advanced information and communication technologies as productivity tool and to provide VR interventions for consumers.

Retention/talent management. Human capital is one of the most valuable intangible assets of any organization. With baby boomers entering retirement, the Generation X (those born between 1965 and 1980) workers are now the backbone of the economy, and Generation Y employees (those born between 1981 and 1998) will in time become the backbone of our labor force (Henson, 2009). State VR agencies must devise effective retention/talent management strategies to attract, retain, and motivate Gen X and Gen Y employees. While work motivation and job satisfaction study is very common in the business world (e.g., Allen \& Meyer, 1990; Schaufeli \& Bakker, 2003), there is a lack of research devoted to examine the relationship among work motivation, workplace stress, burnout, organizational commitment, work engagement, and job satisfaction and life satisfaction of employees in state VR agencies.

It seems that in order to bring state VR service delivery into the 21 st century, a comprehensive research plan developed to study the business models used by state VR agencies, the adaptability of these agencies to use innovations and technologies, and how they recruit, retain, and engage their employees to provide customized and effective services using innovations and advanced information and communication technologies to yield outcomes valued by people with disabilities is warranted.

\subsection{Evidence-based VR best practices}

As mentioned, the evidence-based medicine movement has transfused to influence other health and rehabilitation professions (Chan et al., 2011). We are encouraged to find that state VR agencies in our multistate case studies are implementing EBP to improve educational and employment outcomes for people with disabilities, including secondary transition and the IPS model of supported employment. However, the efficacy of other promising practices identified in the study such as benefits counseling, workplace socialization skills training, and workplace support interventions has not been comprehensively evaluated. It should be noted that he qualitative data collected and analyzed in this initial study of evidence-based and promising practices will be used in the next stage of this investigation in a national Delphi Study of vocational rehabilitation subject matter experts to gain a national consensus on the relative importance and portability of these promising prac- 
tices for other state agencies to implement to increase employment outcomes for those served. Once these data are available and a national consensus is reached through the planned Delphi process, specific interventions and services that appear the most promising can move forward to the state VR agencies through technical assistance, consultation, and training interventions can be developed (intervention development) and validated (intervention efficacy) to help state VR agencies examine and adopt these EBP into the tools available for counselors to utilize to effect positive employment outcomes with the consumers they serve.

Importantly, there are other empirically supported interventions that are not promoted by these high performance state VR agencies. For example, enhancing client motivation has been considered one of the essential ingredients needed to provide effective VR services (Chan, Shaw, McMahon, Koch, \& Strauser, 1997; Cook, 2004). Rehabilitation consumers' lack of motivation can be attributed to feelings of hopelessness and passivity, unrealistic treatment goals, fear of losing social security benefits, and unstable job markets (Manthey, Brooks, Chan, Hedenblad, \& Ditchman, in press; Manthey et al., 2011; Thoreson, Smits, Butler, \& Wright, 1968). Motivation is also affected by internal and external determinants of behavior (Deci \& Ryan, 2002). Specifically, self-determination has been found to have a strong effect on treatment engagement and compliance (Edmunds, Ntoumanis, \& Duda, 2006; $\mathrm{Ng}$ et al., 2012). Both motivational interviewing and self-determination are not well integrated as a major component of the treatment paradigm in VR.

Additionally, emphasizing assets and strengths to promote psychosocial adjustment to disability and well-being is fundamental philosophy to VR service delivery practice (Chou, Chan, Phillips, \& Chan, 2013). The strength-focused approach of rehabilitation research and practice provides a solid foundation for the integration of positive psychology into VR. Positive psychology as the scientific study of positive subjective experience, positive emotions, and positive traits, is enjoying a growing body of research identifying and assessing positive personal traits and other positive psychology constructs (e.g., hope, resilience, and subjective well-being), and a variety of instruments and interventions have been developed and validated for use in positive psychology research and practice that can be used to improve outcomes in VR.

The current emphasis of the federal disability agencies on the use of a data-driven approach to improve employment outcomes of people with dis- abilities underscores the importance of demand-side employment interventions. The use of a dual-customer approach by agencies in our study is an example of how these agencies incorporate innovations to improve performance. However, demand-side employment interventions are not uniformly integrated in rehabilitation services by all state VR agencies.

\subsection{Gaps and research directions in evidence-based VR practices}

The selection and utilization of EBP even in high performance state VR agencies are not systemic or based on careful research of the value of the EBP and needs assessment. Recently, the World Health Organization International Classification of Functioning, Disability, and Health (ICF) model, with its emphasis on the importance of environmental (E) factors and personal characteristic $(\mathrm{P})$ factors and the significant $\mathrm{P} \times \mathrm{E}$ interaction effect on the full integration of individuals with disability into the community, has been embraced by health and rehabilitation researchers to advance their EBP agenda, and the voluminous research generated by researchers worldwide can also be invaluable for rehabilitation counselors. Specifically, the ICF framework is composed of three major constructs: (a) body functions and structure, (b) activities (related to tasks and actions by an individual) and participation (involvement in a life situation), and (c) personal and environmental factors. Functioning and disability are viewed as a complex interaction between the health condition of the individual and the contextual factors of the environment as well as personal factors. The ICF model is consistent with the holistic philosophy of rehabilitation counseling and can be an invaluable framework for case conceptualization purposes and can be used by counselors to determine the need for medical, psychosocial and vocational rehabilitation assessment, plan the consumer's rehabilitation program, and select empirically supported VR interventions for people with chronic illness and disability. Using the ICF model of disability to develop a systematic VR research agenda may allow researchers to quickly identify and validate several universal best practices (e.g., supported employment for ID/DD, IPS model of supported employment for people with severe mental illness, secondary transition, postsecondary life adjustment interventions, motivational interviewing, positive psychology interventions, self-determination interventions, benefits counseling, demand-side employment interventions, and mobile apps interventions) that can be integrated into rehabil- 
itation counselors' repertoire of best practices to treat most of the issues presented by people with disabilities receiving services from state VR agencies.

\section{Knowledge translation}

Knowledge translation (KT) is a complex, multidimensional concept involving various methods, measures, and approaches at both the individual and systemic, or contextual, levels (Sudsawad, 2007). Originally developed and applied within the context of health and healthcare systems, KT is now gaining recognition for applicability across a broader range of professional fields (CIHR, 2005; Sudsawad, 2007; WHO, 2005).

A functional working definition, as proposed by the National Center for the Dissemination of Disability Research (NCDDR), describes KT as, "the collaborative and systematic review, assessment, identification, aggregation, and practical application of high-quality disability and rehabilitation research by key stakeholders (i.e., consumers, researchers, practitioners, and policymakers) for the purpose of improving the lives of individuals with disabilities" (NCDDR, 2005). This definition is appropriate for use by rehabilitation researchers, vocational rehabilitation (VR) counselors and administrators, and policy makers. While the construct of KT may be relatively new, it is gaining recognition as an important complement to research by enhancing the transfer and application of research to best practices.

The Knowledge-to-Action (KTA) framework provides a clear model for $\mathrm{KT}$ in rehabilitation. KTA represents the key components of KT including multidirectional communication, interactive processes, ongoing collaboration among partners, multiple activities, diverse knowledge-user groups, impact-oriented, and emphasis on use of research-generated knowledge (Graham et al., 2006; Sudsawad, 2007).

The concept of bridging the gap between research and practice is particularly important when discussing emerging, promising, and evidence-based practice (EBP). KT processes are inherently bidirectional and participatory in nature, as are the steps necessary to identify and replicate EBP (MacDermid \& Graham, 2009). As identified by the RRTC-EBP VR, the concept of EBP is also relatively new to the state-federal VR system. While emerging and promising practices are being identified, the need to test and validate these practices to further inform and expedite development of evidence-based practices for use by VR practitioners is essential (Del Valle, et al., 2014). Embedding KT methods, approaches, and measurements in future research and program evaluation specific to VR will be imperative for continuing collaborative relationships between researchers and practitioners in an effort to truly transfer research findings into practice and improve programmatic outcomes.

\subsection{Critical need for $K T$ across $V R$ partners/stakeholders}

In recent years, the vocational rehabilitation professionals are faced with increased demand for accountability in rehabilitation practice drawing much attention to evidence-based practice (EBP) (Chan, Bezyak, et al., 2010; Chan, Sung, et al., 2011). Most VR staff value research for practice and are willing to apply ideas generated from research to develop Individualized Plans for Employment (IPE) (Graham et al., 2013). They also believe that further training may help them better engage in applying evidence-based practice (Anderson, Matthews, Lui, Nierenhausen, \& Lawler, 2014). However, VR counselors commonly regard research as difficult to comprehend and generally irrelevant to their practice (Martin \& Martin, 1989; Winch, Henderson, \& Creedy, 2005). With work hours dedicated almost entirely on providing direct case services, time constraints present as a significant barrier (Corrigan, Steiner, McCracken, \& Barr, 2001; O'Donnell, 2004).

The KTA model is a viable solution to addressing the problem of bridging the gap in information flow of effective intervention supported by research findings to practitioners. This will provide practitioners with relevant, timely resources that encourage them to appraise the relevance and utilize data with specific cases (Lui, Anderson, Matthews, Nierenhausen, \& Schlegelmilch, 2014; Murray, 2009). The transfer of research findings should be a circuitous and bidirectional flow of information between the researcher and practitioner to ensure that new evidence-informed interventions are applicable knowledge and grounded in real-world experience (Kerner, 2006). Furthermore, the utilization of participatory approaches to engage the key stakeholders (i.e. consumers/families, educators/researchers, practitioners, administrators, and policy makers) including the employers/business and industry, in the planning, development and implementation of all research, training, dissemination, and technical assistance activities should help to increase the likelihood of system and practice change (Baumbusch et al., 2008). 
The key stakeholder of employers/business and industry (demand) is included because they are vital partners in ensuring successful employment outcomes for individuals with disabilities (supply) (Golden \& Karpur, 2012). The knowledge creation and action process in the Knowledge-To-Action (KTA) Model will help disability service providers develop beneficial relationships with employers and help bridge the gap between the supply and demand while adopting evidence-based practices (Bruyere, Erickson, \& VanLooy, 2006; Shaheen et al., 2011).

\subsection{Future direction of $K T$ within the VR program}

As stated by Sudsawad (2007), KT is a developmental process that needs to be multi-modal, multi-directional, requiring adoption of a "model of actionable feedback" (p.17) to "integrate knowledge generation with knowledge diffusion and utilization" (p.18). The formative evaluation aligns well with continuous quality improvement (CQI) and research-based utilization frameworks. A comprehensive, participatory KT process with ongoing feedback and evaluation presents an opportunity to complete the loop between research, application, practice and outcomes in the state-federal VR system.

The objectives of KT as noted by federal partners, "the multidimensional, active process of ensuring that new knowledge gained through the course of research ultimately improves the lives of people with disabilities, and furthers their participation in society" (U.S. Department of Education, 2006, p. 8195) are aligned with the stated goals of the Rehabilitation Act. Additionally, VR staff nationally report interest in using EBP as well as barriers inhibiting implementation of EBP including limited time, need for interactive and pointin-time training and technical assistance, and practical engagement strategies (Anderson, et al., 2014; Graham et al., 2013). These barriers can appropriately and effectively be addressed by integrating contemporary KT concepts, resources, and measures in all future federally funded research and training projects (Lui et al., 2014). By integrating KT into future research and training centers, the National Institute on Disability and Rehabilitation Research (NIDRR) and the Rehabilitation Services Administration (RSA) are providing strong support for VR administrators and practitioners by not merely endorsing the use of evidence-informed and evidence-based practices, but also providing the functional information and tools necessary for EBP implementation in the field. The goal of providing VR counselors and administrators with practical evidenceinformed and evidence-based tools and point-in-time resources is to improve the lives of people with disabilities and enhance employment outcomes in the VR program nationally.

\section{Conclusion}

The purpose of this qualitative study was to discover emerging and promising vocational rehabilitation service delivery practices that helped improve employment outcomes of people with disabilities. The multi-stage qualitative analysis involved the selection of four high performing state VR agencies (Texas, Utah, Mississippi and Maryland) based on annual adjusted rehabilitation rates and other indicators of innovations in practice. In addition to identifying promising best practices, the study also provided a comprehensive analysis of the policies, procedures, practices and structural elements related to the provision of effective best practices to individuals with disabilities served in the four-state sample of VR agencies that lead to successful employment outcomes.

Although there has been significant amount of research conducted over the past 50 years in vocational rehabilitation, most of this work has been descriptive in nature with many of these efforts attempting to determine relationships among existing data, rather than a purposeful approach to define what types of intervention or services appear to work best with what specific populations, under what specific conditions (Paul, 1967). Clearly, this question is an area of weakness and limitation in regard to our existing research emphasis that needs to be addressed in future research initiatives in order to establish evidence-based practices. As indicated by Saunders, Leahy, McGlynn, and Estrada-Hernandez (2006), we need more intervention related studies (re-experimental, quasi experimental and experimental) and less ad hoc studies regarding employment and other related outcomes of the rehabilitation process. As indicated earlier, research efforts need to be theoretically based, with clear definitions of variables, populations and interventions. Data, such as effect size and confidence intervals, need to be routinely included in research reports to maximize our ability to use meta-analysis and other statistical approaches. Finally, in order to meaningfully address knowledge translation and the development of evidence-based practices, we need more replication and extensions of previous research in order to build coherent, rigorous 
lines of research that serve to specifically inform practice and policy in the future (Fleming, Del Valle, Kim \& Leahy, 2013).

There is no question that moving forward on this developing front will be challenging, and will require a comprehensive, multifaceted, and systemic approach to the problem for VR state agencies and the public rehabilitation program. Rehabilitation researchers need to work very closely with state VR agencies to identify and test out new best practices and the process of evidence-based practice within the agencies. Rehabilitation educators have a critical role to play as well in preparing rehabilitation counselors at the masters level who are knowledgeable about the process of evidencebased practice and highly skilled in using available tools to make clinical decisions that are supported by the best available evidence. At the doctoral level we need to prepare future educators and researchers to acquire sophisticated skills in knowledge generation and transformation in order to provide the research and knowledge translation necessary for effective use by practitioners in the public rehabilitation program and beyond, in making evidenced-based clinical decisions (Leahy \& Arokiasamy, 2010).

We are however encouraged to learn that the high performance VR agencies in the multi-state study have demonstrated they are using some features of the integrative/generative business models to improve client service, increase participation, and support datadriven policy and decision making. They are also using advanced health information technologies to support both back-office processes and front-office innovations to better health outcomes, economic self-sufficiency, and social inclusion. The Harvard University's LNW Human Services Value Curve framework may prove to be a useful framework to guide organizational studies in VR and lessons learned from conducting programmatic research in VR management practices will allow us to develop technical assistance interventions based on universal best practices to help state VR agencies in the country move towards the use of the integrative/generative business models to improve the efficiency and effectiveness of VR agencies. The use of the ICF as a VR framework for case conceptualization and systematic research to increase the number of EBP that can be integrated into a VR professional's repertoire of practices will lead to better outcomes for people with disabilities. Research funding used to support programmatic and theory-, model-, or framework driven research to improve organizational performance and to inform clinical VR practices may prove to be the best strategy to maximize return-on-investment for federal and private-sector investments in VR research.

\section{Acknowledgments}

The contents of this article were developed with support from the Rehabilitation Research and Training Center on Evidence-Based Practice in Vocational Rehabilitation at the University of Wisconsin-Madison and the University of Wisconsin-Stout and with funding provided by the U.S. Department of Education, National Institute on Disability and Rehabilitation Research (Grant H133B100034). The ideas, opinions, and conclusions expressed, however, are those of the authors and do not represent recommendations, endorsements, or policies of the U.S. Department of Education.

\section{References}

American Public Human Services Administration (APHSA) (2013). On the road to a 21st century business model: Where are health and human service organizations today? Washington, DC: Author.

Anderson, C., Matthews, P., Lui, J., Nierenhausen, E., \& Lawler, M. (2014, in review). Engaging vocational rehabilitation counselors in knowledge translation processes: A participatory action approach. Rehabilitation Counselors and Educators Journal.

Atkins, D., \& Giusti, C. (2005). The confluence of poverty and disability. In C. Armbrister \& K. Smith (Eds.), The realities of poverty in Delaware 2003-2004 (pp. 6-8). Dover, DE: Delaware Housing Coalition.

Baumbusch, J. L., Kirkham, S. R., Khan, K. B., McDonald, H., Semeniuk, P., Tan, E. \& Anderson, J. M. (2008). Pursuing common agendas: A collaborative model for knowledge translation between research and practice in clinical settings. Research in Nursing and Health, 31, 130-140.

Bolton, B., \& Akridge, R. L. (1995). A meta-analysis of skills training programs for rehabilitation clients. Rehabilitation Counseling Bulletin, 38, 262-273.

Brault, M. W. (2012). Americans with disabilities: 2010 (Current Population Report No. P70-131). Washington D.C.: U.S. Census Bureau.

Bruyere, S. M., Erickson, W. A., \& VanLooy, S. A. (2006). The impact of business size on employer ADA response. Rehabilitation Counseling Bulletin, 49(4), 194-206.

Campbell, K., Bond, G. R., \& Drake, R. E. (2011). Who benefits from supported employment: A meta-analytic study. Schizophrenia Bulletin, 37, 370-380.

Canadian Institutes of Health Research (CIHR). (2005). About knowledge translation. Retrieved from http://www.cihrirsc.gc.ca/e/29418.html

Chan, F., Bezyak, J., Ramirez, M., Chiu, C., Sung, C., \& Fujikawa, M. (2010). Concepts, challenges, barriers, and opportunities related 
to evidence-based practice in rehabilitation counseling. Rehabilitation Education, 24, 179-190.

Chan, F., Shaw, L. R., McMahon, B. T., Koch, L., \& Strauser, D. (1997). A Model for Enhancing Rehabilitation CounselorConsumer Working Relationships. Rehabilitation Counseling Bulletin, 41(2), 122-137.

Chan, F., Strauser, D., Maher, P., Lee, E.J., Jones, R., \& Johnson, E. T. (2010). Demand-side factors related to employment of people with disabilities: A survey of employers in the Midwest region of the United States. Journal of Occupational Rehabilitation, 4, 412-419.

Chan, F., Sung, C., Muller, V., Wang, C. C., Fujikawa, M., \& Anderson, C. A. (2011). Evidence-based practice and research utilization. In D. Maki, \& V. M. Tarvydas (Eds.), Professional practice of rehabilitation counseling (pp. 391-412). New York: Springer Publishing Company.

Chan, F., Tarvydas, V., Blalock, K., Strauser, D., \& Atkins, B. (2009). Unifying and elevating rehabilitation counseling through model-driven, culturally-sensitive evidence-based practice. Rehabilitation Counseling Bulletin, 52(2), 114-119.

Chou, C. C., Chan, F., Chan, Y. C., \& Phillips, B. (2013). Introduction to positive psychology in rehabilitation. Rehabilitation Research, Policy, \& Education, 27, 126-130.

Corrigan, P. W., Steiner, L., McCracken, S. G., Blaser, B., \& Barr, M. (2001). Strategies for disseminating evidence-based practices to staff who treat people with serious mental illness. Psychiatric Services, 52, 1598-1606

Dean, D., Pepper, J., Schmidt, R., \& Stern, S. (2013). The effects of vocational rehabilitation for people with cognitive impairments. Retrieved from http://econweb.umd.edu/ $\sim$ davis/eventpapers/SternEffects.pdf

Dean, D., Pepper, J., Schmidt, R., \& Stern, S. (2014). State vocational rehabilitation programs and federal disability insurance: An analysis of Virginia's vocational rehabilitation program. IZA Journal of Labor Policy, 3, 7. Retrieved from http://www. izajolp.com/content/pdf/2193-9004-3-7.pdf

Deci, E. L., \& Ryan, R. M. (1985). Intrinsic motivation and selfdetermination in human behavior. New York: Plenum.

Del Valle, R., Leahy, M. J., Sherman, S., Anderson, C., Tansey, T. N., \& Schoen, B. (2014). Promising best practices that lead to employment in vocational rehabilitation: Findings from a fourstate multiple case study. Journal of Vocational Rehabilitation, 41(2), 99-113.

Ditchman, N., Wu, M., Chan, F., Fitzgerald, S., Lin, C. P., \& Tu, W. (2013). Vocational rehabilitation. In D. Strauser (Ed.), Career development, employment, and disability in rehabilitation: From theory to practice (pp. 343-360). New York: Springer Publishing Company.

Dutta, A., Gervey, R., Chan, F., Chou, C. C., \& Ditchman, N. (2008). Vocational rehabilitation services and employment outcomes of people with disabilities: A United States study. Journal of Occupation Rehabilitation, 18, 326-334.

Edmunds, J., Ntoumanis, N., \& Duda, J. L. (2006). A test of selfdetermination theory in the exercise domain. Journal of Applied Social Psychology, 36, 2240-2265.

Fleming, A. R., Del Valle, R., Kim, M., \& Leahy, M. J. (2013). Best practice models of effective vocational rehabilitation service delivery in the public rehabilitation program: A review and synthesis of the empirical literature. Rehabilitation Counseling Bulletin, 56(3), 146-159.
Fogg, N., Harrington, P., \& McMahon, B. (2011). The underemployment of persons with disabilities during the Great Recession. The Rehabilitation Professional, 19(1), 3-10.

Golden, T., \& Karpur, A. (2012) Translating knowledge through blended learning: A comparative analysis of face-to-face and blended learning methods. Rehabilitation Research Policy, and Education, 26(4), 305-314.

Graham, G., Inge, K., Wehman, P., Murphy, K., Revell, W. G., \& West, M. (2013). Moving employment research into practice: Knowledge and application of evidence-based practices by state vocational rehabilitation agency staff. Journal of Vocational Rehabilitation, 39, 75-81.

Graham, I. D., Logan, J., Harrison, M. B., Straus, S. E., Tetroe, J., Caswell, W., \& Robinson, N. (2006). Lost in knowledge translation: Time for a map? The Journal of Continuing Education in the Health Professions, 26(1), 13-24.

Henson, R. (2009). $21^{\text {st }}$ century human capital management: Adapting to the demands of change (An Oracle White Paper). Redwood City, CA: Oracle Corporation. Retrieved from http://www. oracle.com/us/products/applications/ebusiness/21-century-hcmrow-henson-396487.pdf

Horvath, A. O., Del Re, A., Flückiger, C., \& Symonds, D. (2011). Alliance in individual psychotherapy. In J. C. Norcross (Ed.), Psychotherapy relationships that work (2nd ed., pp. 25-69). New York: Oxford University Press.

Kaye, S. H. (2010). The impact of the 2007-09 recession on workers with disabilities. Monthly Labor Review, 133(10), 19-30.

Kerner, J. F. (2006). Knowledge translation versus knowledge integration: A funder's perspective. The Journal of Continuing Education in the Health Professions, 26(1), 72-80.

Krause, J. S., Carter, R. Pickelsimer, E., \& Wilson, D. (2008). A prospective study of health and risk of mortality after spinal cord injury. Archives of Physical Medicine and Rehabilitation, 89, 1482-1491.

Leahy, M. J. \& Arokiasamy, C. V. (2010). Prologue: Evidence-based practice research and knowledge translation in rehabilitation counseling. Rehabilitation Education, 24(3\&4), 173176.

Leahy, M., Chan, F., Sung, C., \& Kim, M. (2013). Empirically derived test specifications for the certified rehabilitation counselor examination. Rehabilitation Counseling Bulletin, 56, 199-214.

Leahy, M. J., Del Valle, R. J., Sherman, S. G., Anderson, C., Tansey, T., Lui, J., et al. (2013). Phase II final report: Multiple case studies on effective vocational rehabilitation services delivery practices. Lansing, MI: Rehabilitation Research and Training Center on Effective VR Service Delivery Practices.

Leahy, M. J., Thielsen, V. T., Millington, M. J., Austin, B., \& Fleming, A. (2009). Quality assurance and program evaluation: Terms, models, and applications in rehabilitation administration. Journal of Rehabilitation Administration, 33(2), 69-82.

Lui, J., Anderson, C. A., Matthews, P., Nierenhausen, E., \& Schlegelmilch, A. (2014). Knowledge translation strategies to improve the resources for rehabilitation counselors to employ best practices in the delivery of vocational rehabilitation services. Journal of Vocational Rehabilitation, 41(2), 137-145.

MacDermid, J. C., \& Graham, I. D. (2009). Knowledge Translation: Putting the "Practice" in Evidence-Based Practice, Hand Clinics, 25(1), 125-143.

Manthley, T, Brooks, J., Chan, F., Hedenblad, L., \& Ditchman, N. (in press). Motivational interviewing in rehabilitation health settings. 
In F. Chan, N. Berven, \& K. Thomas (Eds.), Counseling theories and techniques for rehabilitation health professionals (2nd ed,). New York: Springer Publishing Company.

Manthey, T., Jackson, C., \& Evans-Brown, P. (2011). Motivational interviewing and vocational rehabilitation: A review with recommendations for administrators and counselors. Journal of Applied Rehabilitation Counseling, 42(1), 3-14.

Martin, D., \& Martin, M. (1989). Current trends: Bridging the gap between research and practice. Journal of Counseling \& Development, 87, 108-116.

Martin, R., West-Evans, K., \& Connelly, J. (2010). Vocational rehabilitation: Celebrating 90 years of careers and independence. American Rehabilitation, 15-18.

Murali, V., \& Oyebode, F. (2014). Poverty, social inequality and mental health. Advances in Psychiatric Treatment, 10, 216-224

Murray, C. E. (2009). Diffusion of innovation theory: A bridge for the research-practice gap in counseling. Journal of Counseling and Development, 87, 108-116.

National Center for the Dissemination of Disability Reserch (2005). What is knowledge translation? Focus (Technical Brief No. 10). Retrieved from http://www.ktdrr.org/ ktlibrary/articles_pubs/ncddrwork/focus/focus10/Focus10.pdf

Ng, J. Y., Ntoumanis, N., Thøgersen-Ntoumani, C., Deci, E. L., Ryan, R. M., Duda, J. L., \& Williams, G. C. (2012). Self-determination theory applied to health contexts: A meta-analysis. Perspectives on Psychological Science, 7, 325-340.

O'Donnell, C. A. (2004). Attitudes and knowledge of primary care professionals towards evidence-based practice: A postal survey. Journal of Evaluation in Clinical Practice, 10, 197-205.

Prochaska, J. O., DiClemente, C. C., \& Norcross, J. C. (1992). In search of how people change. American Psychologist, 47, 1102-1114.

O'Neil, J., Mamun, A., Potamites, E., Chan, F., \& Cardoso, E. (2014). Return to work of SSDI beneficiaries who do and don't access state vocational rehabilitation agency services: Case control study. West Orange, NJ: Kessler Foundation, Rehabilitation Research and Training Center on Individual Characteristics.

Paul, L. (1967). Strategy of outcome research in psychotherapy. Journal of Counseling Psychology (31), 109-118.

Rehabilitation Services Administration (RSA). (2008). FY2008 evaluation standards and performance indicators for the vocational rehabilitation services program. Washington, D.C.: Author. Retrieved from http://www2.ed.gov/rschstat/eval/rehab/2008indicators/index.html

Rogers, E. (2003). Diffusion of innovation (5th ed.). New York: The Free Press.

Rosenthal, D. A., Hoyt, W. T., Ferrin, J. M., Miller, S., \& Cohen, N. D. (2006). Advanced methods in meta-analytic research: Applications and implications for rehabilitation counseling research. Rehabilitation Counseling Bulletin, 49(4), 234-246.

Saez, E. (2013). Striking it richer: The evolution of top incomes in the United States (Updated with 2012 preliminary estimates). Berkeley, CA: Department of Economics, University of California.

Saunders, J. L., Leahy, M. J., McGlynn, C., \& Estrada-Hernandez, N. (2006). Predictors of employment outcomes for persons with disabilities: An integrative review of potential evidenced-based factors. Journal of Applied Rehabilitation Counseling, 37(2), 3-20.

Shaheen, G., Golden, T. P., Dowse, P., Myhill, W. N., Hill, E. L., Hoff, D.,... Kenney, C. (2011). Transformation of center-based work into integrated opportunities for people with disabilities: Analysis and recommendation for center-based employers. Ithaca, NY: Cornell University Employment and Disability Institute. Retrieved from http://www.nymakesworkpay.org/rpmsheltered.cfm

Sherman, S., Leahy, M. J., Del Valle, R., Anderson, C., Tansey, T. N., \& Lui, J. (2014). Organizational and cultural factors that promote creative best practices in the public rehabilitation program: Findings from a four-state multiple case study. Journal of Vocational Rehabilitation, 41(2), 115-125.

Substance Abuse and Mental Health Services Administration. (2009). Supported employment: How to use the evidence-based practices KITs (DHHS Pub. No. SMA-08-4364). Rockville, MD: Author. Retrieved from http://store.samhsa.gov/shin/content//SMA084365/HowtoUseEBPKITS-SE.pdf

Sudsawad, P. (2007). Knowledge translation: Introduction to models, strategies, and measures. Austin, TX: Southwest Educational Development Laboratory, National Center for the Dissemination of Disability Research.

Technology and Entrepreneurship Center at Harvard. (2010). The next generation of human services: Realizing the vision (A Report from the 2010 Human Services Summit at Harvard University). Retrieved from http://community.Inwprogram. org/sites/default/files/Realizing_the_Vision.pdf

Thoreson, R. W., Smits, S. J., Butler, A. J., \& Wright, G. N. (1968). Counselor problems associated with client characteristics (Wisconsin studies in vocational rehabilitation). Madison: University of Wisconsin Regional Rehabilitation Research Institute.

U.S. Department of Education (2006). The National Institute on Disability and Rehabilitation Research-Notice of final long-range plan for fiscal years 2005-2009, Federal Register, 31, 81658200 .

U.S. Department of Labor (2014). Office of Disability Employment Policy-Current disability employment statistics. Retrieved from http://www.dol.gov/odep/

U.S. Department of Labor (2012a). Economic News Release: Table A-6. Employment status of the civilian population by sex, age, and disability status, not seasonally adjusted. Retrieved from: http://www.bls.gov/news.release/empsit.t06.htm

U.S. Department of Labor (2012b). Economic News Release: Table 1. Employment status of the civilian noninstitutionalized population by disability and selected characteristics. Retrieved from: http://www.bls.gov/news.release/disabl.t01.htm

U.S. Department of Labor (1991). What work requires of schools? A Secretary's Commission on Achieving Necessary Skills (SCANS) report for America 2000. Washington, DC: Author. Retrieved from http://wdr.doleta.gov/SCANS/whatwork/whatwork.pdf

U.S. Government Accountability Office (2005). Vocational Rehabilitation-Better measures and monitoring could improve the performance of the VR Program (GAO-05-865). Washington, DC: Author.

de Veer, A. J. E., Fleuren, M. A. H. Bekkema, N. \& Francke, A. L. (2011). Successful implementation of new technologies in nursing care: A questionnaire survey of nurse-users. BMC Medical Informatics and Decision Making, 11(67), 1-12. Retrieved from http://www.biomedcentral.com/content/pdf/1472-6947-1167.pdf

Wampold, B. E. (2001). The great psychotherapy debate. Mahwah, NJ: Erlbaum.

Wehman, P., Chan, F., Ditchman, N., \& Kang, H. J. (2014). Effect of supported employment on vocational rehabilitation outcomes 
of transition-age youth with intellectual and developmental disabilities: A case control study. Intellectual and Developmental Disabilities, 52, 296-310.

Winch, S., Henderson, A., \& Creedy, D. (2005). Read, think, do!: A method for fitting research evidence into practice. Journal of Advanced Nursing, 50, 20-26.
World Health Organization (WHO) (1995). Bridging the gaps. Geneva, Switzerland: Author.

World Health Organization (WHO) (2005). Bridging the "Know-Do" gap: Meeting on knowledge translation in global health. Retrieved from http://www.who.int/kms/WHO_ EIP_KMS_2006_2.pdf 